\title{
Balanço de Energia nas Condições de Mudanças de Uso do Solo na Região Sul do Estado de Mato Grosso
}

\author{
Lucas Peres Angelini ${ }^{1}$, Pablinne Cynthia Batista Silva e Silva ${ }^{2}$, Marcos Alves Fausto $^{2}$, \\ Nadja Gomes Machado ${ }^{2}$, Marcelo Sacardi Biudes ${ }^{3}$ \\ ${ }^{1}$ Campus Rio Verde, Instituto Federal Goiano, Rio Verde, GO, Brasil. \\ ${ }^{2}$ Programa de Pós-Graduação em Física Ambiental, Instituto de Física, \\ Universidade Federal de Mato Grosso, Cuiabá, MT, Brasil. \\ ${ }^{3}$ Campus Cuiabá-Bela Vista, Instituto Federal de Mato Grosso, Cuiabá, MT, Brasil.
}

Recebido em 22 de Setembro de 2016 - Aceito em 17 de Abril de 2017

\begin{abstract}
Resumo
A conversão da vegetação nativa para áreas agrícolas modifica a dinâmica de troca de energia no sistema soloplanta-atmosfera. Dessa forma, o objetivo deste estudo foi avaliar o efeito da conversão de uma área de Cerrado em culturas agronômicas no balanço de energia e evapotranspiração no sul do Estado de Mato Grosso. O estudo foi conduzindo em áreas de cerradão, cerrado stricto sensu, mangueiral, cultura de teca, coqueiral e pastagem. O balanço de energia foi estimado pelo SEBAL (Surface Energy Balance Algorithm for Land), utilizando dados de estação meteorológica e imagens do sensor TM Landsat 5 obtida em 2010. O saldo de radiação apresentou menores valores em pastagem que em Cerrado. O fluxo de calor latente no Cerrado foi maior em até $60 \%$ com relação às culturas agronômicas. O fluxo de calor sensível nas áreas agrícolas foram 50\% maior que áreas de Cerrado. O Cerradão e o mangueiral apresentaram menores valores de fluxo de calor no solo. Apesar desse estudo não representar todo o Cerrado de Mato Grosso, os resultados demonstraram o impacto da conversão do Cerrado em áreas agrícolas no balanço de energia.

Palavras-chave: imagens de satélite, mudança do uso do solo, desmatamento.
\end{abstract}

\section{Energy Balance in Terms of Land Use Changes in the Southern of Mato Grosso State}

\begin{abstract}
The conversion of native vegetation to agricultural areas modifies the dynamics of energy exchange in the soil-plantatmosphere system. Thus, the objective of this study was to evaluate the effect in energy balance and evapotranspiration in the southern state of Mato Grosso due to the conversion of a Cerrado area in agronomic crops. The study was leading in areas of Cerradão, Cerrado strictu sensu, mango grove, teak culture, coconut trees and pasture. The energy balance was estimated by SEBAL (Surface Energy Balance Algorithm for Land) using weather station data and images from Landsat 5 TM sensor obtained in 2010. The net radiation showed lower values in pasture than in the Cerrado. The latent heat flux in the Cerrado was higher by $60 \%$ in relation to agronomic crops. The sensible heat flux in agricultural areas were 50\% higher than Cerrado areas. The Cerradão and Mangueiral showed lower heat flux values on the ground. Although this study does not represent the entire Cerrado of Mato Grosso, the results showed the impact of the Cerrado into agricultural areas in the energy balance.
\end{abstract}

Keywords: satellite images, land use change, deforestation.

\section{Introdução}

O Cerrado, segundo maior bioma brasileiro, é reconhecido como a savana mais rica do mundo, abrigando uma flora com mais de 11.000 espécies de plantas nativas (Mendonça et al., 2008), das quais 4.400 são endêmicas (Myers et al., 2000). Destaca-se que, os principais tributários das

Autor de correspondência: Marcelo Sacardi Biudes, marcelo@fisica.ufmt.br. 
bacias Amazônica e do São Francisco nascem em áreas de Cerrado. Com apenas $7,44 \%$ de sua área protegida por unidades de conservação, o Cerrado já apresenta aproximadamente $50 \%$ de sua área nativa convertida em lavouras e pastagem (MMA, 2011).

O desmatamento no estado de Mato Grosso tem aumentado a cada ano e com ele a produção agrícola (Fearnside, 2003; Azevedo e Saito, 2013). De acordo com CONAB (2014), Mato Grosso é o segundo estado brasileiro em produção agrícola e responde por $24 \%$ da produção de grãos no Brasil, somando 187 milhões de toneladas. Entre as culturas com maior destaque estão a soja $(24,7 \%)$, a cana-de-açúcar (19,8\%) e o milho (13,2\%) (IBGE, 2014). No entanto, o desmatamento desenfreado provoca diversos danos climáticos em escala regional.

Sabe-se que, o desmatamento modifica o albedo e a temperatura da superfície, influenciando as trocas de energia entre a superfície e a baixa atmosfera (Sheil e Murdiyarso, 2009). Consequentemente, o desmatamento afeta a umidade ar, a estabilidade térmica da camada limite e o regime de chuvas em larga escala (Hasler e Avissar 2007; Costa e Pires, 2010). Portanto, a quantificação dos componentes do balanço de energia em larga escala nas condições de mudança de uso do solo é fundamental para compreender as interações solo-planta-atmosfera e melhorar os modelos climáticos regionais, especialmente em áreas onde a água é limitante (Fischer et al., 2008).

A quantificação da mudança no balanço de energia nas condições de munda de uso da terra são quase ou totalmente desconhecidas em largas escalas espaciais sobre diferentes tipos de cobertura do solo no Cerrado matogrossense (Biudes et al., 2015). Em geral, técnicas micrometeorológicas são utilizadas para medir os padrões temporais dos componentes do balanço de energia em superfícies vegetadas em experimento de longa duração. No entanto, as medidas realizadas por essas técnicas apresentam alto custo de instalação e manutenção. (Allen et al., 2007). As técnicas micrometeorológicas representam condições pontuais, em áreas heterogêneas e extensas o seu uso é restrito, falhando em análises a nível de escalas hidrográficas (Fausto et al., 2014).

Neste sentido, a determinação dessas medidas em áreas heterogêneas com diferentes tipos de cobertura do solo e grandes extensões tem sido possível, com precisão comprovada e baixo custo, através do uso de imagens de satélites ambientais e/ou meteorológicos. Como exemplo, na região sul do estado de Mato Grosso, a estimativa do balanço radiativo utilizando sensoriamento remoto foi validada usando dados de torres micrometeorológica na Bacia do Alto Rio Paraguai (Fausto et al., 2014) e no norte do Pantanal Mato-Grossense (Danelichen et al., 2013; Biudes et al., 2014). Para se estimar os componentes do balanço de energia em escala regional com imagens de satélite, o SEBAL (Surface Energy Balance Algorithm for Land) é a técnica mais popular que tem sido amplamente utilizada em diferentes países sob diferentes tipos de cobertura (Bastiaanssen et al., 2005; Allen et al., 2011).

A vantagem do algoritmo SEBAL é a utilização de poucos dados de superfície como temperatura, umidade relativa do ar e velocidade do vento (da Silva et al., 2014). No entanto, tem havido dificuldade em validar os componentes do balanço de energia com dados medidos na superfície (Bezerra et al., 2008).

Considerando que, a economia do estado de Mato Grosso é baseada na agropecuária e que técnicas de sensoriamento remoto podem estimar em escala regional os efeitos da mudança do uso do solo no balanço de energia, o objetivo deste estudo foi avaliar o efeito da conversão de uma área de Cerrado em culturas agronômicas no balanço de energia no sul do Estado de Mato Grosso.

\section{Material e Métodos}

\section{1. Área de estudo}

A área de estudo está localizada na Fazenda Experimental da Universidade Federal de Mato Grosso (UFMT) (1547'11" S-5604'47” O), no município de Santo Antônio de Leverger, distante à $33 \mathrm{~km}$ de Cuiabá, Mato Grosso, Brasil (Fig. 1). As áreas selecionadas para este estudo foram: mangueiral (A), Cerradão (B), Cerrado Stricto Sensu (C), Cultura de teca (D), Coqueiral (E) e Pastagem (F).

De acordo com a classificação de Köppen-Geiger, o clima regional é Aw com precipitação anual de $1335 \mathrm{~mm}$, com uma estação chuvosa de outubro a abril e uma seca de maio a setembro (Chiaranda et al., 2012) e temperatura média anual entre $24{ }^{\circ} \mathrm{C}$ e $26^{\circ} \mathrm{C}$ (Biudes et al., 2012). A vegetação nativa remanescente é constituída por fragmentos de Cerrado stricto sensu e Cerradão. A vegetação do Cerrado Stricto sensu é caracterizada por árvores de porte médios em torno de $3 \mathrm{~m}$ de altura, enquanto que no Cerradão as árvores chegam a atingir mais de $10 \mathrm{~m}$ de altura (Klink, 2005).

\subsection{Instrumentação utilizada}

Os dados meteorológicos de entrada do SEBAL dispostos na Tabela 1 foram obtidos por um termohigrômetro (HMP45AC, Vaisala, Inc., Woburn, MA, USA), um anemômetro (014A Met-One Wind Speed Sensor, Campbell Scientific), um piranômetro (LI200X, LI-COR Biosciences, Inc., Lincoln, NE, USA). A precipitação para caracterização pluviométrica no período de estudo foi medida por um pluviômetro de báscula (TB4, Campbell Scientific, Inc., Logan, Utah). Os equipamentos conectados em uma bateria eram alimentados por um painel solar (SP65, Campbell Scientific, Inc., Logan, UT, USA), com 65 W de potência. Os dados coletados foram processados e armazenados por um datalogger (CR 10X, Campbell Scientific, Inc., Logan, Utah), com médias de $15 \mathrm{~min}$. 

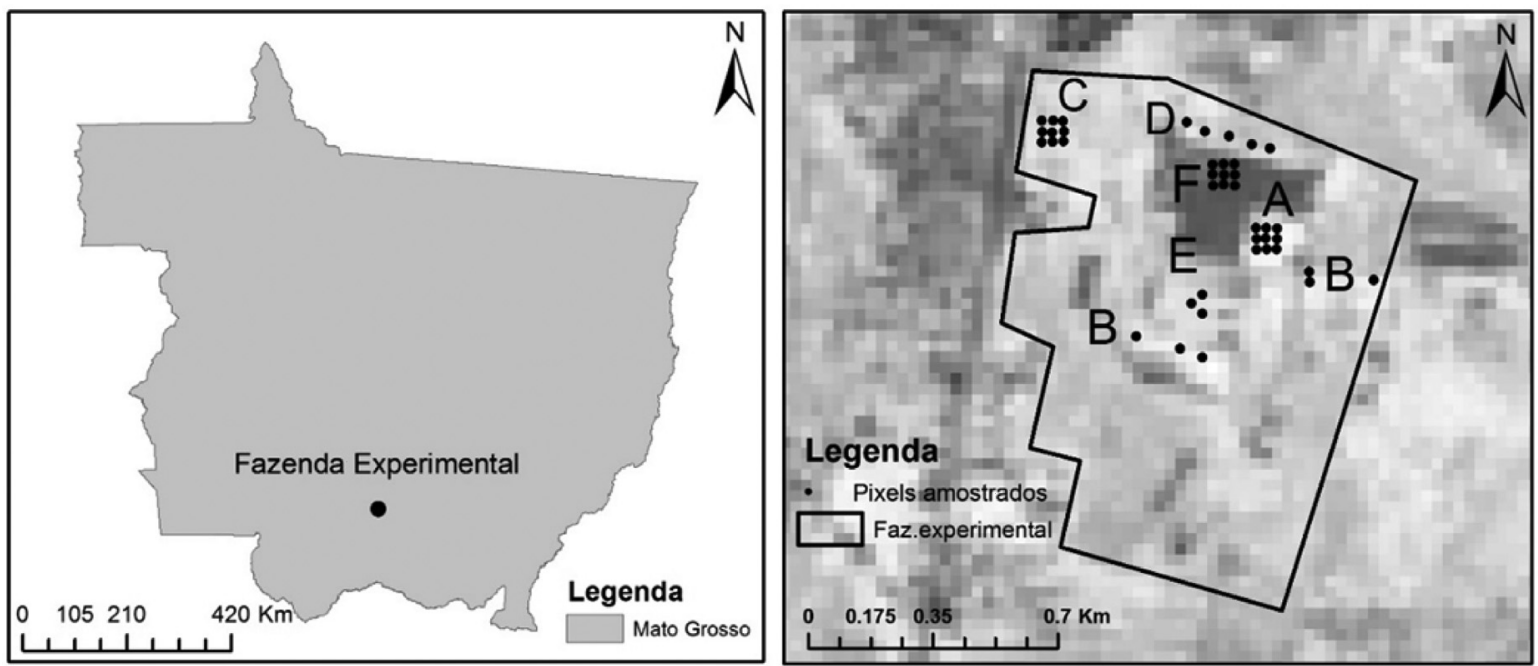

Figura 1 - Localização das áreas de mangueiral (A), Cerradão (B), Cerrado Stricto Sensu (C), cultura de teca (D), coqueiral (E) e pastagem (F) na Fazenda Experimental da Universidade Federal de Mato Grosso em Santo Antônio do Leverger-MT.

Tabela 1 - Valores da temperatura do ar (Tar), umidade relativa do ar (UR), pressão atual de vapor d'água (ea), velocidade do vento $(u)$, pressão atmosférica (Patm) e radiação solar incidente $(R g)$ no momento da passagem do satélite Landsat 5.

\begin{tabular}{|c|c|c|c|c|c|c|}
\hline Data & $\operatorname{Tar}\left({ }^{\circ} \mathrm{C}\right)$ & UR (\%) & $e_{a}(\mathrm{kPa})$ & $u(\mathrm{~m} / \mathrm{s})$ & Patm (kPa) & $R g\left(\mathrm{~W} \mathrm{~m}^{-2}\right)$ \\
\hline $18 / 03 / 2010$ & 31,2 & 59 & 2,67 & 1,79 & 99,7 & 763,9 \\
\hline $19 / 04 / 2010$ & 30,5 & 60 & 2,61 & 2,00 & 99,7 & 692,6 \\
\hline 06/06/2010 & 21,9 & 65 & 1,71 & 2,23 & 100,3 & 586,1 \\
\hline $22 / 06 / 2010$ & 27,4 & 61 & 2,23 & 1,88 & 99,9 & 559,2 \\
\hline $24 / 07 / 2010$ & 26,3 & 60 & 2,06 & 2,01 & 99,9 & 589,1 \\
\hline $12 / 10 / 2010$ & 32,3 & 35 & 1,68 & 2,00 & 99,6 & 861,5 \\
\hline
\end{tabular}

\subsection{Dados orbitais utilizados}

As imagens utilizadas neste estudo foram obtidas pelo sensor Thematic Mapper (TM) Landsat 5 da órbita 226 e ponto 71 nos dias 18/03, 19/04, 06/06, 22/06, 24/07 e 12/10 de 2010. As imagens de refletância da superfície corrigidas para o efeito da atmosfera foram obtidas na plataforma Earth Explorer do Serviço Geológico dos Estados Unidos, disponível em [http://earthexplorer.usgs.gov/]. A temperatura da superfície foi corrigida para efeitos da atmosfera utilizando uma equação linear múltipla em função da emissividade da superfície e da temperatura da superfície não corrigida, estimada pela atenuação da banda termal pela atmosfera com auxílio do software MODTRAN 5.0 (Barsi et al., 2005).

Para áreas de Cerrado Stricto Sensu, Pastagem e Mangueiral foram extraídos valores de 09 pixels. Para as demais áreas em função de suas formas irregulares de plantio foram extraídos seis pixels para área de Cerradão, três pixels para área de Coqueiral e cinco pixels para o plantio de Teca.

\subsection{Componentes do balanço de energia}

O balanço de energia (Eq. (1)) representa a soma da energia utilizada na evaporação da água, aquecimento do ar e do solo.

$$
R n=L E+H+G
$$

em que $R n$ representa o saldo de radiação ( $\mathrm{W} \mathrm{m}^{-2}$ ) (Eq. (2)), $L E$ o fluxo de calor latente $\left(\mathrm{W} \mathrm{m}^{-2}\right), H$ o fluxo de calor sensível $\left(\mathrm{W} \mathrm{m}^{-2}\right)$ e $G$ é o fluxo do calor no solo $\left(\mathrm{W} \mathrm{m}^{-2}\right)$.

$$
R n=R_{S \downarrow}\left(1-\alpha_{\text {sup }}\right)-R_{L \uparrow}+R_{L \downarrow}-\left(1-\varepsilon_{0}\right) R_{L \downarrow}
$$

em que $R_{S \downarrow}$ é a radiação de ondas curtas incidente, medida na estação meteorológica instalada na área de estudo, $\alpha_{s u p}$ é o albedo da superfície de cada pixel (Eq. (3); Liang et al., 2002), $R_{L \downarrow}$ é a radiação de ondas longas emitida pela atmosfera na direção de cada pixel, $R_{L} \uparrow$ é a radiação de ondas longas emitida por cada pixel e $\varepsilon_{0}$ é a emissividade de cada pixel.

$$
\begin{aligned}
& \alpha_{\text {sup }}=0,356 \rho_{1}+0,130 \rho_{3}+0,373 \rho_{4}+ \\
& 0,085 \rho_{5}+0,072 \rho_{7}-0,0018
\end{aligned}
$$


em que $\rho_{1}, \rho_{3}, \rho_{4}, \rho_{5}$, e $\rho_{7}$ são as refletâncias da superfície das bandas 1, 3, 4, 5 e 7 do TM Landsat 5. Tentativas experimentais de utilizar as 6 bandas para estimar o albedo da superfície resultou em desvio padrão muito elevado para a banda 2, sendo que sua exclusão não representou mudança significativa nas estimativas (Liang et al., 2000).

As $R_{L \uparrow}$ e $R_{L \downarrow}$ (Eqs. (4) e (5)) foram calculadas pela equação de Stefan-Boltzman (Allen et al., 2002).

$$
\begin{aligned}
& R_{L \uparrow}=\varepsilon_{0} \cdot \sigma \cdot T_{S}^{4} \\
& R_{L \downarrow}=\varepsilon_{a} \cdot \sigma \cdot T_{a}^{4}
\end{aligned}
$$

em que $\varepsilon_{0}$ é a emissividade da superfície de cada pixel, $\varepsilon_{a}$ é a emissividade da atmosfera de cada pixel, $\sigma$ é a constante de Stefan-Boltzman $\left(\sigma=5,67.10^{-8} \mathrm{~W} \mathrm{~m}^{-2} \mathrm{~K}^{-4}\right)$ e $T_{s}$ e $T_{a}$ são a temperatura da superfície e do ar $(\mathrm{K})$, respectivamente.

A $\varepsilon_{0}$ foi calculada pela Eq. (6) baseado no modelo de cobertura da vegetação (Valor e Caselles, 1996).

$$
\begin{aligned}
& \varepsilon_{0}=\varepsilon_{v} P_{v}+\varepsilon_{g}\left(1-P_{v}\right)\left(1-1,74 P_{v}\right)+ \\
& 1,7372 P_{v}\left(1-P_{v}\right)
\end{aligned}
$$

em que $\varepsilon_{v}$ e $\varepsilon_{g}$ são as emissividade da vegetação do solo puro, usados valores de 0,985 e 0,960 (Valor e Caselles, 1996), respectivamente, e $P_{v}$ é a fração de cobertura da vegetação calculado pela Eq. (7) em função do NDVI (Normalized Difference Vegetation Index; Eq. (8)).

$$
P_{v}=\left[\frac{\left(N D V I-N D V I_{\text {min }}\right)}{\left(N D V I_{\max }-N D V I_{\text {min }}\right)}\right]^{2}
$$

em que $N D V I_{\max }$ e $N D V I_{\min }$ são os valores de $N D V I$ máximos e mínimos da área de estudo e $\rho_{4}$ e $\rho_{3}$ são as refletâncias da superfície das bandas 4 e 3 do TM Landsat 5 .

A emissividade da atmosfera foi calculada em função da $T_{a}$ e da pressão de vapor d'água do $\operatorname{ar}\left(e_{a}\right)$ obtidos da estação meteorológica no momento da passagem do satélite (Eq. (8)) (Duarte et al., 2006).

$$
\varepsilon_{a}=0,625\left(\frac{e_{a}}{T_{a}}\right)^{0,131}
$$

O fluxo de calor no solo foi calculado pela Eq. (9) (Bastiaanseen, 2000).

$$
G=\left[T_{S}\left(0,0038 a_{\text {sup }}+0,0074 a_{\text {sup }}{ }^{2}\right)\left(1-0,98 N D V I^{4}\right)\right] R n
$$

em que $T_{s}$ é temperatura de superfície, $a_{\text {sup }}$ é albedo de superfície, $N D V I$ é índice de vegetação por diferença normalizada e $R n$ é saldo de radiação.

$\mathrm{O} H$ é um parâmetro fundamental no cálculo do balanço de energia no algorítimo SEBAL. Sua estimativa foi realizada com base em um processo interativo em que o comprimento de Monin-Obukhov $L$ foi utilizado para obter-se funções de correções que estabilizam os valores da resistência aerodinâmica $\left(r_{a \mathrm{~h}}\right)$ (Eq. (10)). Esse processo leva em consideração a velocidade do vento e um gradiente de temperatura de superfície segundo expressa Bastiaanssen et al. (1998).

$$
H=\rho c_{p} \frac{\left(a+b T_{S}\right)}{r_{a h}}
$$

em que $\rho$ é a densidade específica do ar, $c_{p}$ é o calor específico do ar a pressão constante $\left(1004 \mathrm{~J} \mathrm{~kg}^{-1} \mathrm{~K}^{-1}\right), r_{a h}$ é a resistência aerodinâmica ao transporte de calor sensível $\left(\mathrm{sm}^{-1}\right)$ (Eq. (11)), admitindo-se a condição de estabilidade atmosférica (Bastiaanseen, 1998; Allen et al., 2002; Bezerra et al., 2008).

$$
r_{a h}=\frac{\ln \left(\frac{z_{2}}{z_{1}}\right)}{u_{*} \cdot k}
$$

em que $z_{1}$ e $z_{2}$ são as alturas em metros acima da superfície (Geralmente considera-se entre 0,1 e 2,0 m), $u_{*}$ é a velocidade de fricção $\left(\mathrm{m} \mathrm{s}^{-1}\right), k$ é a constante de von Karman $(0,41)$.

Para se calcular a $u_{*}$ (Eq. (13)) foi necessário definir o coeficiente de rugosidade ao transporte de momentum do local $\left(z_{o m}\right)$ dado em função do SAVI (Soil Adjusted Vegetation Index) Eq. (12) (Bastiaanssen et al., 2000).

$$
\begin{aligned}
& z_{\text {om }}=\exp (-5,809+5,62 \mathrm{SAVI}) \\
& u_{*}=\frac{k u_{200}}{\ln \left(\frac{z_{200}}{z_{\text {om }}}\right)}
\end{aligned}
$$

em que $z_{200}$ é a velocidade do vento estimada na altura de 200 metros.

A determinação de $u_{200}$ ocorre a níveis da atmosfera, onde o efeito da rugosidade da superfície não influencia a velocidade do vento (blending height). Admitindo-se essas condições, a $u_{200}$ foi calculada pela Eq. (14) (da Silva et al., 2014).

$$
u_{200}=\frac{u_{z} \ln \left(\frac{200}{z_{\text {om }}}\right)}{\ln \left(\frac{z}{z_{\text {om }}}\right)}
$$

em que $u_{z}$ é a velocidade do vento medida na estação meteorológica $\left(\mathrm{ms}^{-1}\right)$.

Para estimar a diferença de temperatura $(d T)$ utilizou-se as informações dos pixels ancoras em uma relação linear com a $T_{S}$ (Eq. (16); Allen et al., 2002).

$$
d T=a+b T_{S}
$$

em que $a$ e $b$ são coefícientes obtidos da diferença da temperatura entre dois níveis $z_{1}$ e $z_{2}$ de pixels denominados 
"ancoras", sendo um "frio" onde se admite que há ausência de calor sensível, e um "quente", onde se admite que há ausência de calor latente.

Os valores de $H$ estimados serviram como valores iniciais no processo interativo, onde foi aplicado a correção de estabilidade atmosférica pelo comprimento de MoninObukhov $(L)$ em função do fluxo de calor sensível, velocidade de fricção e temperatura de superfície de cada pixel (Eq. (16)).

$$
L=\frac{\rho C_{p} u_{*}^{3} T_{S}}{K g H}
$$

em que $g$ é a aceleração da gravidade terrestre, sendo $H$ estimado considerando a condição de neutralidade da atmosfera. Para $L<0$, a atmosfera é considerada instável, se $L>0$, a atmosfera é considerada estável e se $L=0$ a atmosfera é considerada neutra.

O fluxo de calor latente diário $\left(L E_{24 h}\right)$ foi calculado por meio da Eq. (17).

$$
L E_{24 \mathrm{~h}}=F E_{i} R n_{24 \mathrm{~h}}
$$

em que $F E_{i}$ é a fração evaporativa instantânea (Eq. (18)) e $R n_{24 \mathrm{~h}}$ é o saldo de radiação diário $\left(\mathrm{W} \mathrm{m}^{-2}\right)$ (Eq. 19).

$$
\begin{aligned}
& F E_{i}=\left(\frac{L E}{R n-G}\right)=F E_{24 \mathrm{~h}}=\frac{L E_{24 \mathrm{~h}}}{R n_{24 \mathrm{~h}}} \\
& R n_{24 \mathrm{~h}}=R s_{24 \mathrm{~h}}\left(1-a_{\text {sup }}\right)-123 \tau_{24 \mathrm{~h}}
\end{aligned}
$$

em que $F E_{24 \mathrm{~h}}$ é a fração evaporativa diária, admitida igual à fração evaporativa instantânea $F E_{i}$ (Allen et al., 2011; Bastiaanssen et al., 2000), Rn - $G$ é a energia disponível instantânea $\left(\mathrm{W} \mathrm{m}^{-2}\right) ; R s_{24 \mathrm{~h}}$ é a radiação solar incidente diária $\left(\mathrm{W} \mathrm{m}^{-2}\right)$ e $\tau_{24 \mathrm{~h}}$ é a transmissividade diária da atmosfera (Allen et al., 2002). O coeficiente 123 tem sido amplamente utilizando para a estimativa da $R n_{24 \mathrm{~h}}$, para área de Cerrado, esse coeficiente tem se mostrado satisfatório, resultando em valores de $E T_{24 \mathrm{~h}}$ consistente com dados medi- dos (da Silva et al., 2014). Para outros sistemas é recomendado verificações com medidas em campo.

A evapotranspiração diária $\left(E T_{24 \mathrm{~h}}\right)$ foi estimada pela Eq. (20).

$$
E T_{24 \mathrm{~h}}=\frac{\left(L E_{24 \mathrm{~h}} \times R n_{24 \mathrm{~h}} \times 86,4\right)}{2450} .
$$

\section{Resultados e Discussão}

A precipitação apresentou um total acumulado de $1100 \mathrm{~mm}$ entre os meses de janeiro a março e de outubro a dezembro. A estação seca ficou concentrada nos meses de abril a setembro com acumulado total de $150 \mathrm{~mm}$ (Fig. 2). A tendência sazonal da precipitação na área de estudo foi compatível com a climatologia regional (Biudes et al., 2015). A região durante a estação seca apresentou índices pluviométricos abaixo dos $100 \mathrm{~mm}$ mensal, o que é consistente com a duração da estação seca na região (Danelichen et al., 2013; Biudes et al., 2014).

A variação do NDVI foi mais expressiva na pastagem, contudo, não houve diferença significativa do NDVI entre a estação seca e chuvosa nas áreas de Cerrado e de culturas agronômicas. Na estação chuvosa, o NDVI em pastagem aumentou em média $70 \%$ em relação a estação seca (Fig. 3). Os maiores valores de NDVI na estação chuvosa é justificando pela disponibilidade de água no sistema, o que aumenta a biomassa na superfície (Cunha et al., 2012; Martins et al., 2015). O NDVI é o indicador de verdor da vegetação e sua diminuição está relacionado ao estresse hídrico (Fu e Burgher, 2015).

O NDVI na pastagem apresentou padrão diferente em relação as áreas de Cerradão e Cerrado Stricto sensu e de culturas agronômicas, pois os maiores valores ocorreram na estação seca, fato esse justificado pela irrigação da área durante esse período. No entanto, a variabilidade do NDVI foi maior na estação chuvosa em relação a estação seca. Dentre as culturas, o NDVI do Mangueiral foi o que apre-

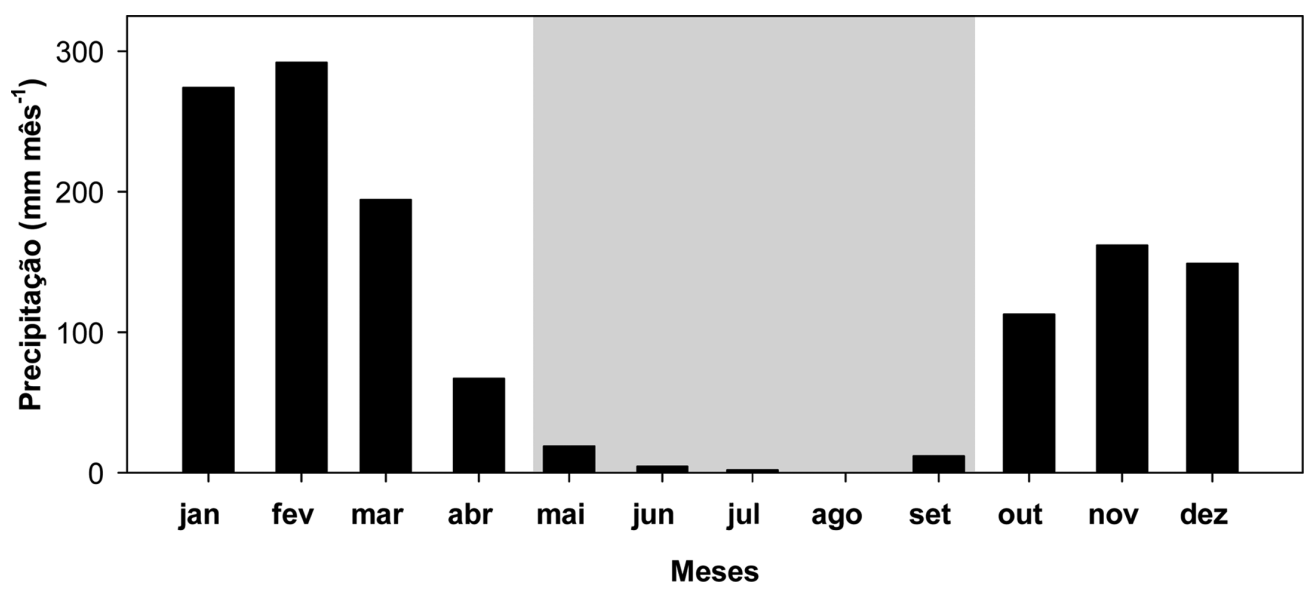

Figura 2 - Precipitação mensal em 2010 na Fazenda Experimental da Universidade Federal de Mato Grosso em Santo Antônio do Leverger-MT. Área sombreada representa a estação seca. 


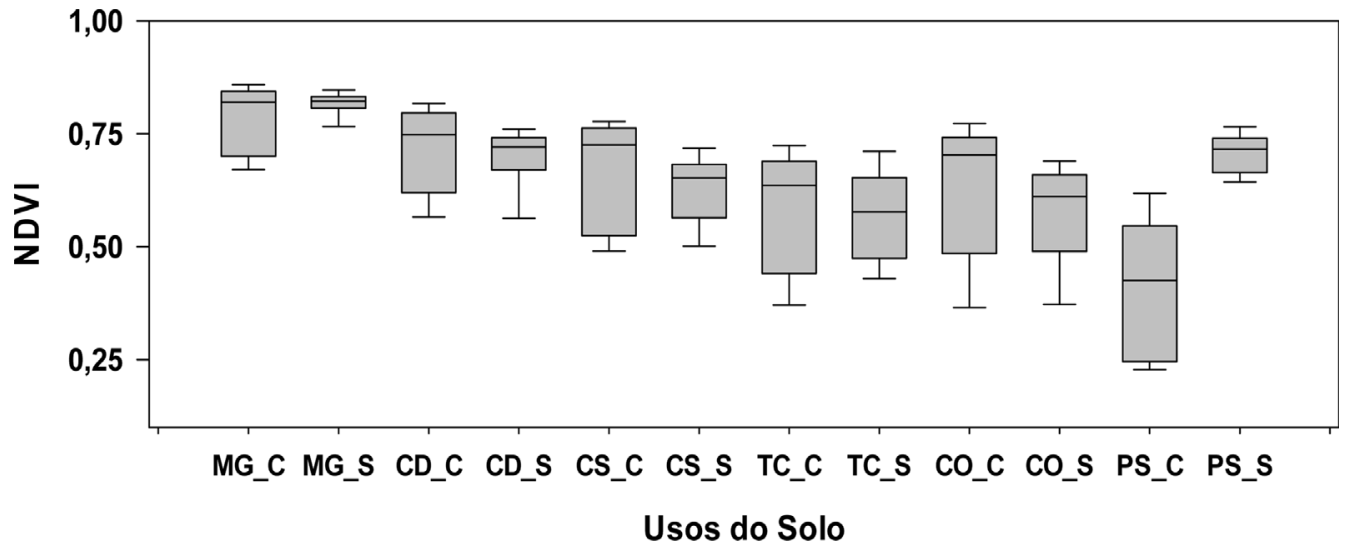

Figura 3 - Normalized Difference Vegetation Index (NDVI) do mangueiral (MG), Cerradão (CD), Cerrado Stricto Sensu (CS), cultura de Teca (TC), coqueiral (CO) e pastagem (PS) durante a estação chuvosa (_C) e seca (_S) na Fazenda Experimental da Universidade Federal de Mato Grosso em Santo Antônio do Leverger-MT.

sentou a menor variação entre as estações, sendo $16 \%$ maior em relação as áreas de Cerrado (Fig. 3).

$\mathrm{O}$ saldo de radiação $(R n)$ foi diferente entre as estações, sendo $41 \%$ maior na estação chuvosa. As áreas de Cerradão e a pastagem tiveram os maiores e os menores valores de $R n$ respectivamente. Na pastagem os valores de $R n$ foram 16\% inferiores em relação ao Cerradão (Fig. 4A). Áreas de floresta possuem maior capacidade de absorção da radiação de onda curta, resultado da complexa e heterogênea arquitetura do dossel (dos Santos et al., 2011), que permite maiores valores de umidade no solo, o que explica os maiores valores de $R n$ no Cerradão. A variabilidade do $R n$ entre a estação seca e chuvosa indica forte influência da sazonalidade da radiação solar, e da influência da cobertura de nuvens e da umidade do solo (Figs. 5A e 5B). Os valores de $R n$ do presente estudo são semelhantes aos encontrados por Fausto et al. (2014) e Biudes et al. (2015) para a mesma localidade.

O fluxo de calor no solo instantâneo $(G)$ foi maior na estação chuvosa (Fig. 4B). Nessa estação ocorreu a maior variabilidade de $G$ nas áreas de Cerrado, pois algumas espécies perdem a cobertura foliar devido ao estresse hídrico, e com o início das chuvas, inicia-se o processo de brotação, aumentando gradativamente a biomassa na superfície (Biudes et al., 2014a).

$\mathrm{O}$ mangueiral e a pastagem tiveram os menores e os maiores valores de $G$ respectivamente. Na pastagem, houve diferença de até $49 \%$ de $G$ em relação as áreas de Cerradão na estação chuvosa, para o mangueiral houve uma redução de até $31 \%$ de $\mathrm{G}$ na estação seca em relação as áreas de Cerrado. (Fig. 4B). Na Pastagem, a variabilidade dos parâmetros biofísicos pode estar relacionada ao rodízio dos pastos para alimentação bovina, o que pode contribuir para o aumento dos valores de $G$ e $H$ na estação chuvosa.

Houve diferença no fluxo de $L E$ entre as estações do ano para o Mangueiral e Cerradão, com maior variabilidade na estação chuvosa. As demais culturas agronômicas e o
Cerrado Stricto sensu não apresentaram diferença significativa do $L E$ entre a estação seca e chuvosa. A pastagem foi o ecossistema com maior variação de $L E$ com valores menores em até $63 \%$ em relação a áreas de Cerrado (Fig. 4C). Na cultura de Teca e Coqueiro, houve redução de $36 \%$ e $26 \%$ do $L E$ em relação a áreas de Cerrado, respectivamente. (Fig. 4C).

Com relação aos valores do $H$ não ocorreram diferenças significativas entre a estação seca e chuvosa nas áreas de Cerrado e de cultura agronômica, excetuando-se na pastagem, onde ocorreu a maior variabilidade de $H$ na estação chuvosa. Na Pastagem, o $H$ foi até $71 \%$ maior em relação as áreas de Cerrado. Para as culturas de Teca e Coqueiro houve incremento de $44 \%$ e $29 \%$ de $H$ em relação as áreas de Cerrado, respectivamente. Os menores valores de $H$ foram encontrados no Mangueiral (Fig. 4D).

As áreas de Cerrado tiveram os maiores valores de $E T$ na estação chuvosa com relação à estação seca. Com exceção da Pastagem, os valores de ET diferiram significativamente entre as estações seca e chuvosa (Fig. 4E). De acordo com a Fig. 4E, a pastagem apresentou a maior variabilidade de $E T$ na estação chuvosa, enquanto que a menor variabilidade de ET ocorreu no Mangueiral, indicando menor efeito da sazonalidade no Mangueiral em relação a pastagem, áreas de Cerrado, Teca e na cultura de coco.

A ET na pastagem foi inferior em até $63 \%$ em relação as áreas de Cerrado. Para as culturas de Teca e Coqueiro os valores de ET foram inferiores em até $31 \%$ e $23 \%$ em relação as áreas de Cerrado. Na estação seca, os valores de ET no mangueiral foram semelhantes aos de Cerradão e Cerrado Stricto Sensu (Fig. 4E). Mesmo tendo o maior NDVI, o Mangueiral evapotranspirou até $15 \%$ menos do que as áreas de Cerrado. A espacialização da ET pode ser vista nas Figs. 5C e 5D.

As espécies do Cerrado possuem alta rugosidade aerodinâmica, alta capacidade de extração de água de regiões mais profundas da solo e grande quantidade biomassa 


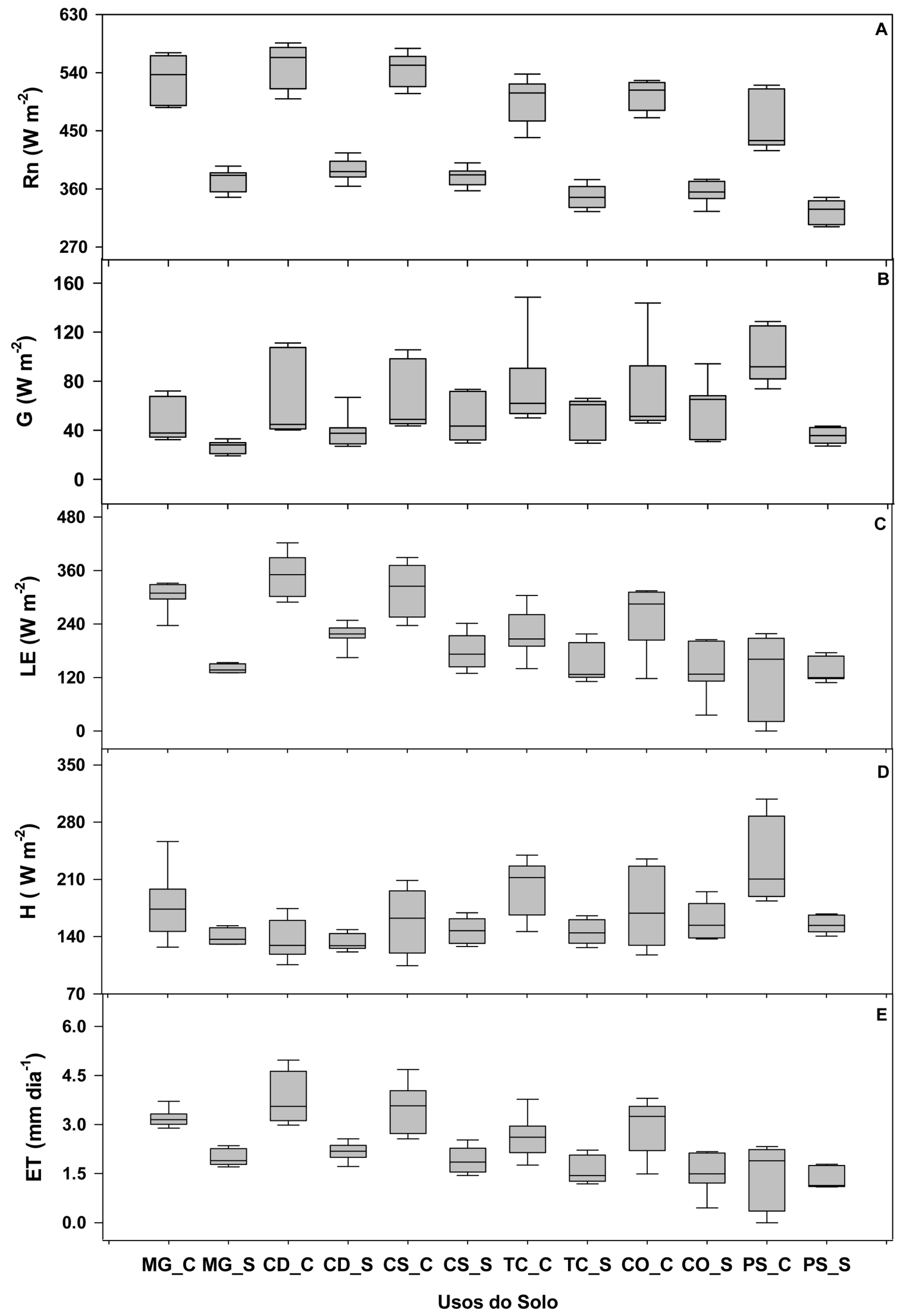

Figura 4 - Saldo de radiação $(R n, \mathrm{~A})$, fluxo de calor no solo $(G, \mathrm{~B})$, fluxo de calor latente $(L E, \mathrm{C})$, fluxo de calor sensível $(H$, D) e evapotranspiração diária (ET, E) do mangueiral (MG), Cerradão (CD), Cerrado Stricto Sensu (CS), cultura de Teca (TC), coqueiral (CO) e pastagem (PS) durante a estação chuvosa (_C) e seca (_S) na Fazenda Experimental da Universidade Federal de Mato Grosso em Santo Antônio do Leverger-MT. 


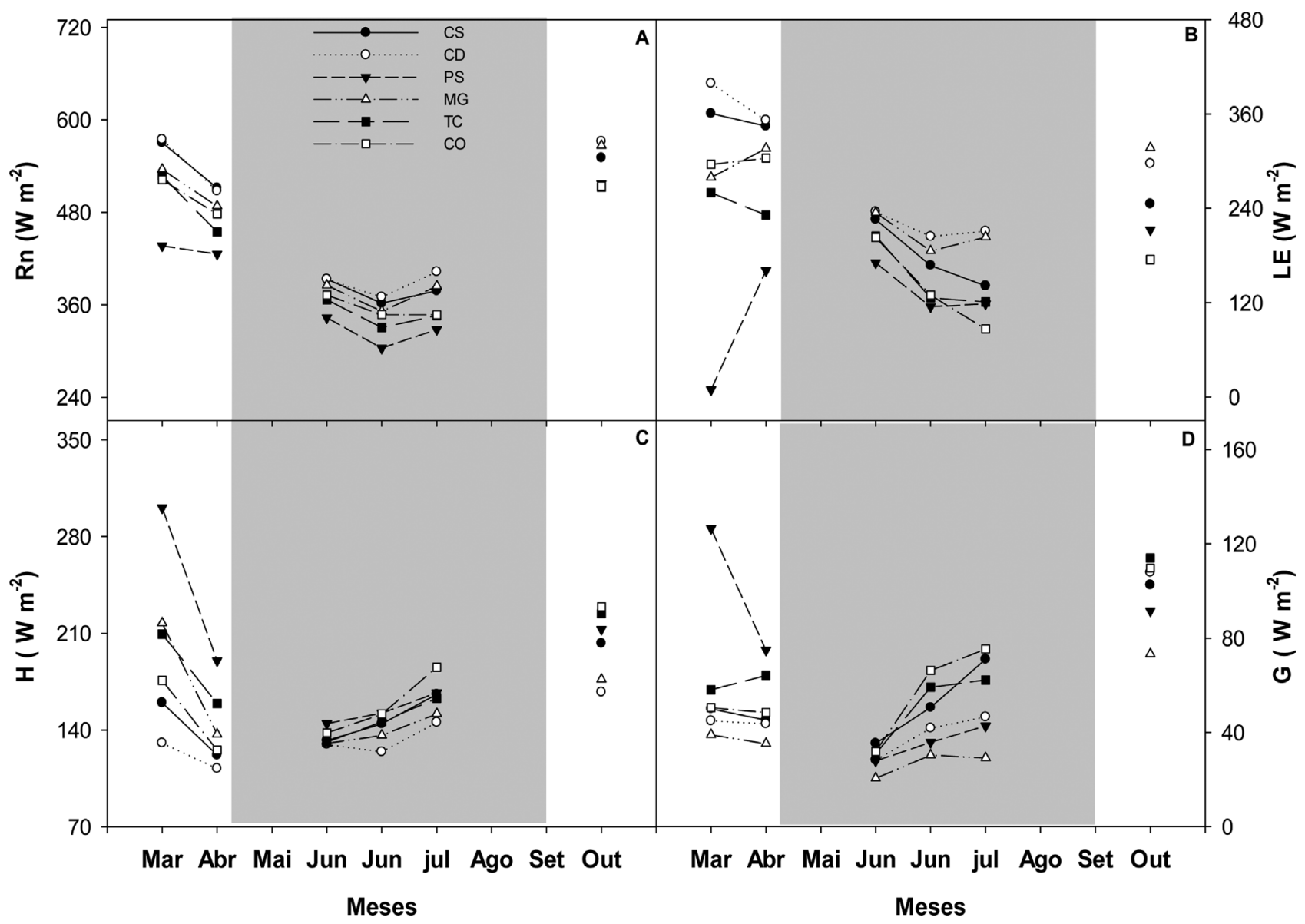

Figura 5 - Cartas de saldo de radiação instantâneo $(R n)$ na estação chuvosa (A) e na estação seca (B) e evapotranspiração diária $(E T)$ na estação chuvosa (C) e na estação seca (D) no ano de 2010 na Fazenda Experimental da Universidade Federal de Mato Grosso - Santo Antônio do Leverger - MT.

(Brannstrom et al., 2008; Biudes et al., 2009), Além dos fatores mencionados, a heterogeneidade do dossel de florestas de Cerrado proporciona maior troca de água no sistema planta-atmosfera (Betts et al., 2008).

A conversão de áreas de Cerrado em áreas de culturas exóticas e pastagem impactam o balanço de radiação e energia no sistema solo-planta-atmosfera (Biudes et al., 2012; Lathuillière et al., 2012). Nas áreas de Cerradão e
Cerrado Stricto Sensu, os valores de Rn, $L E$ e $E T$ diferiram significativamente entre a estação seca e chuvosa.

Os maiores valores de $R n, L E, H$ e $G$ instantâneos foram encontrados na estação chuvosa e os menores na seca. Para os ecossistemas amostrados nesse estudo, o menor $R n$ ocorreu no mês de junho e o maior em outubro (Fig. 6A). O maior particionamento de $R n$ foi em fluxos de $L E$ (Fig. 6B), seguido de $H$ (Fig. 6C) e $G$ (Fig. 6D). Enquanto a fração da energia disponível para $L E$ diminuiu

Tabela 2 - Distribuição do saldo de radiação $(R n)$ em fluxos de calor latente $(L E / R n)$, sensível $(H / R n)$ e no solo $(G / R n)$ nas áreas de Cerradão, Cerrado Stricto Sensu, coqueiral, manqueiral, pastagem e cultura de teca na Fazenda Experimental da Universidade Federal de Mato Grosso em Santo Antônio do Leverger-MT.

\begin{tabular}{|c|c|c|c|c|c|c|c|c|c|}
\hline \multirow[t]{2}{*}{ Área de estudo } & \multicolumn{3}{|c|}{ Anual } & \multicolumn{3}{|c|}{ Chuvosa } & \multicolumn{3}{|c|}{ Seca } \\
\hline & $L E / R n$ & $H / R n$ & $G / R n$ & $L E / R n$ & $H / R n$ & $G / R n$ & $L E / R n$ & $H / R n$ & $G / R n$ \\
\hline Cerradão & 59,7 & 29,5 & 10,9 & 55,8 & 34,2 & 10 & 63,5 & 24,7 & 11,8 \\
\hline Stricto Sensu & 52,7 & 34,3 & 13 & 58,4 & 29,5 & 12,1 & 47,0 & 39,1 & 13,9 \\
\hline Coqueiral & 45,2 & 39,8 & 15,1 & 51,4 & 34,8 & 13,7 & 38,9 & 44,7 & 16,4 \\
\hline Mangueiral & 56,6 & 35,3 & 8,2 & 57,6 & 33,3 & 9,1 & 55,5 & 37,4 & 7,2 \\
\hline Pastagem & 34,1 & 49,6 & 16,2 & 27 & 51,6 & 21,4 & 41,2 & 47,7 & 11 \\
\hline Cultura de Teca & 43,9 & 40,9 & 15,3 & 44,7 & 39,5 & 15,8 & 43,0 & 42,3 & 14,8 \\
\hline
\end{tabular}




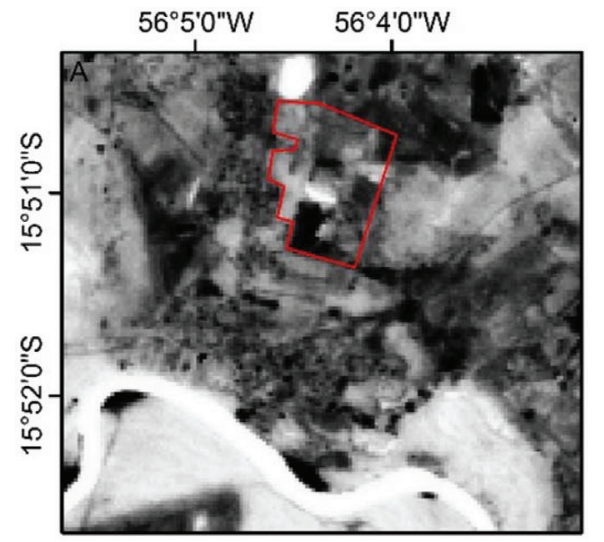

Rn (Wm-2)

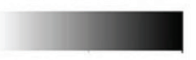

827

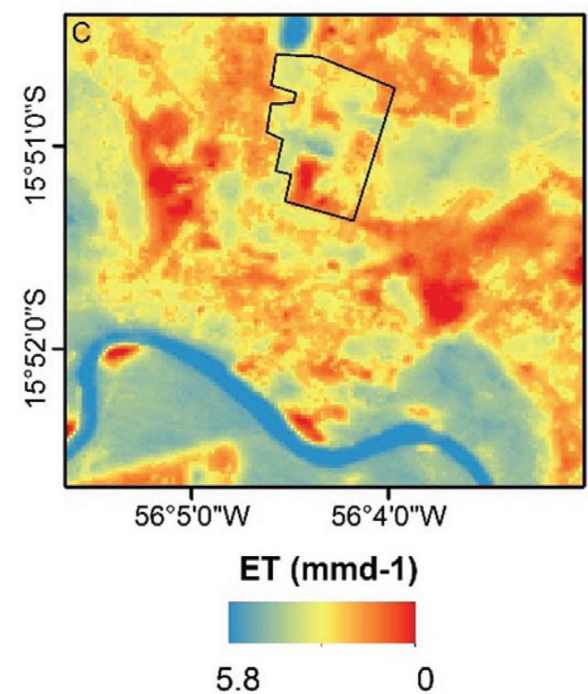

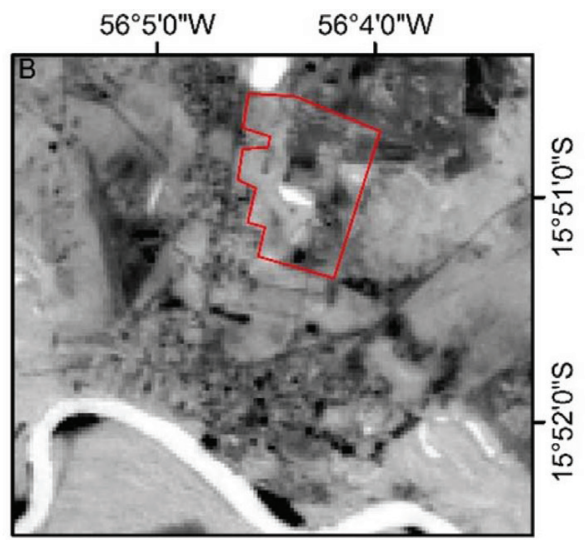

Rn (Wm-2)
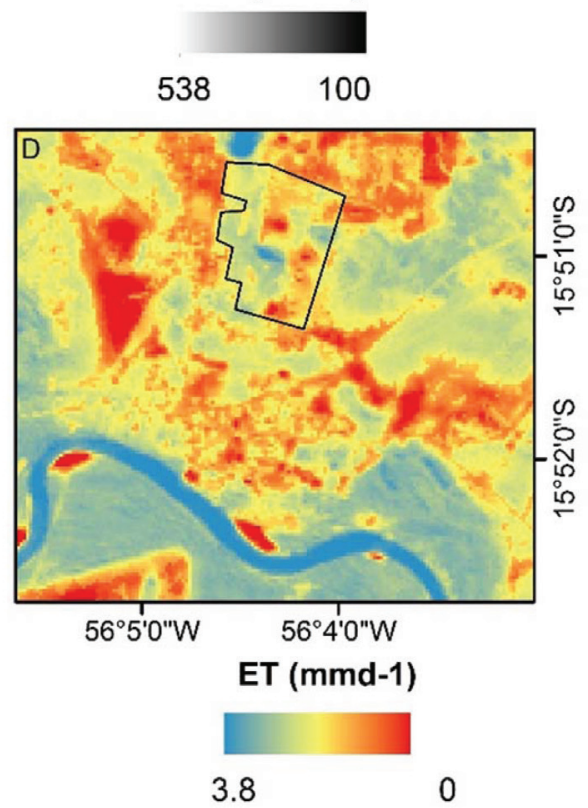

Figura 6 - Variação temporal do saldo de radiação (Rn, A), fluxo de calor latente $(L E, \mathrm{~B})$, fluxo de calor sensível $(H, \mathrm{C})$ e fluxo de calor no solo $(G$, D) instantâneos do Cerrado Stricto Sensu (CS), Cerradão (CD), pastagem (PS), mangueiral (MG), cultura de Teca (TC), coqueiral (CO) ao longo do ano de 2010 na Fazenda Experimental da Universidade Federal de Mato Grosso em Santo Antônio do Leverger-MT. Área sombreada representa a estação seca.

nos meses mais secos, o $H$ aumentou devido a diminuição do NDVI. As áreas de Cerrado utilizam com melhor eficiência a energia disponível em fluxo de $L E$ (Biudes et al., 2015; Dias et al., 2015).

As áreas com maiores valores de NDVI apresentaram as maiores taxas de particionamento de $R n$ em $L E$. Consequentemente, as áreas com menor $N D V I$ apresentaram os maiores valores de $H$ e $G$. A diminuição do NDVI aumenta a parcela de energia para o aquecimento do ar e do solo para a estações seca e chuvosa anualmente (Biudes et al., 2015) (Tabela 2).

\section{Conclusões}

A conversão de áreas de Cerrado em áreas de culturas agronômicas impactou o balanço de radiação e energia na área de estudo, com redução do fluxo de calor latente e evapotranspiração de até $60 \%$, aumento do calor sensível de até $50 \%$ e aumento do fluxo de calor no solo de até $49 \%$ em relação as áreas de Cerrado. Os resultados produzidos nesse estudo foram consistentes com os trabalhos encontrados na literatura cientifica.

\section{Agradecimentos}

A pesquisa foi apoiada pela Universidade Federal de Mato Grosso (UFMT), Programa de Pós-Graduação em Física Ambiental (PPGFA/IF/UFMT), Instituto Federal de Mato Grosso (IFMT), Conselho Nacional de Desenvolvimento Científico e Tecnológico $\left(\mathrm{CNPq}\right.$, processo $\mathrm{n}^{\circ}$ 303625/2015-5; Edital Universal 01/2016, processo $\mathrm{n}^{\circ}$ 407463/2016-0) e Fundação de Amparo à Pesquisa do Estado de Mato Grosso (FAPEMAT - PRONEM 2014, processo $\left.\mathrm{n}^{\circ} 561397 / 2014\right)$. 


\section{Referências}

ALLEN, R.; IRMAK, A.; TREZZA, R. HENDRICKX, J.M.H. Satellite-based ET estimation in agriculture using SEBAL and METRIC. Hydrological Processes, v. 25, n. 26, p. 4011-4027, 2011.

ALLEN, R.; BASTIAANSSEN, W.; WATERS, R.; TASUMI, M.; TREZZA, R. Surface energy balance algorithms for land (SEBAL), Idaho implementation - Advanced training and user's manual, version 1.0, 2002.

ALLEN, R.G.; TASUMI, M.; MORSE, A.; TREZZA R.; WRIGHT, J.L. et al. Satellite-based Energy Balance for Mapping Evapotranspiration with Internalized Calibration, (METRIC) - Applications. Journal of Irrigation and Drainage Engineering, v. 133, n. 4, p. 395-406. 2007.

AZEVEDO, A.A.; SAITO, C.H.O. Perfil dos desmatamentos em Mato Grosso, após implementação do licenciamento ambiental em propriedades rurais. Cerne, v. 19, n. 1, p. 111-122, 2013.

BARSI, J.A.; SCHOTT, J.R.; PALLUCONI, F.D.; HOOK, S.J. Validation of a Web-Based Atmospheric Correction Tool for Single Thermal Band Instruments. Earth Observing Systems X, Proc. SPIE, v. 5882, 2005.

BASTIAANSEEN, W.G.; MENENTI, M.; FEDDES, R.A.; HOLTSLAG, A.A.M. A Remote Sensing Surface Energy Balance Algorithm for Land (SEBAL) 1. Formulation. Journal of Hydrology, v. 212-213, p. 198-212, 1998.

BASTIAANSSEN, W.G.M.; MOLDEN, D.J.; MAKIN, I.W. Remote sensing for irrigated agriculture: examples from research and possible applications. Agricultural Water Management. v. 46, n. 2, p. 137-155, 2000.

BASTIAANSSEN, W.G.M.; NOORDMAN, E.J.M.; PELGRUM, H.; DAVIDS, G.; THORESON, B.P. et al. SEBAL model with remotely sensed data to improve water resources management under actual field conditions. Journal of Irrigation and Drainage Engineering, v. 131, n. 1, p. 85-89, 2005.

BETTS, R.; SANDERSON, M.; WOODWARD, S. Effects of large-scale Amazon forest degradation on climate and air quality through fluxes of carbon dioxide, water, energy, mineral dust and isoprene. Philosophical transactions of the royal society B, v. 393, p. 1873-1880, 2008.

BEZERRA, B.G.; SILVA, B.B.; FERREIRA, N.J. Estimativa da evapotranspiração real diária utilizando-se imagens digitais TM - Landsat 5. Revista Brasileira de Meteorologia, v. 23, n. 3, p. 305-317, 2008.

BIUDES, M.S.; CAMPELO JUNIOR, J.H.; NOGUEIRA, J.S.; SANCHES, L. Estimativa do balanço de energia em cambarazal e pastagem no norte do Pantanal pelo método da razão de Bowen. Revista Brasileira de Meteorologia, v. 24, n. 2, p. 135-143, 2009.

BIUDES, M.S.; NOGUEIRA, J.S.; DALMAGRO, H.J.; MACHADO, N.G.; DANELICHEN, V.H.M. et al. Mudança no microclima provocada pela conversão de uma floresta de cambará em pastagem no Norte do Pantanal. Revista de Ciências Agro-Ambientais, v. 10, n. 1, p. 61-68, 2012.

BIUDES, M.S; MACHADO, N.G. DANELICHEN, V.H.M. SOUZA, M.C. VOURLITIS, G.L. et al. Ground and remote sensing-based measurements of leaf área index in a transitional forest and seasonal flooded forest in Brazil. International Journal of Biometeorology, v. 58, n. 6, p. 11811193, 2014.
BIUDES, M.S.; SOUZA, M.C.; MACHADO, N.G.; DANELICHEN, V.H.M.; VOURLITIS, G.L. et al. Modelling gross primary production of a tropical semi-deciduous forest in the southern Amazon Basin. International Journal of Remote Sensing, v. 35, n. 4, p. 1540-1562, 2014.

BIUDES, M.S.; VOURLITIS, G.L.; MACHADO, N.G.; ARRUDA, P.H.Z.; NEVES, G.A.R. et al. Patterns of energy exchange for tropical ecosystems across a climate gradient in Mato Grosso, Brazil. Agricultural and Forest Meteorology, v. 202, n. 15, p. 112-124, 2015.

BRANNSTROM, C.; JEPSON, W.; FILIPPI, A.M.; REDO, D.; $\mathrm{XU}, \mathrm{Z}$. et al. Land change in the Brazilian Savanna (Cerrado), 1986-2002: Comparative analysis and implications for land-use policy. Land Use Policy, v. 25, p. 579-595, 2008.

CHIARANDA, R.; RIZZI, N.E.; COLPINI, R.; SOARES, T.S.; SILVA, V.S.M. Análise da precipitação e da vazão da bacia do Rio Cuiabá. Revista Brasileira de Ciências Agrárias, v. 7, n. 1, p. 117-122, 2012

CONAB, Companhia Nacional de Abastecimento. Indicadores da Agropecuária, setembro 2014. Brasília, DF, 2014. 98p. Disponível em: http://www.conab.gov.br/OlalaCMS/ uploads/arquivos/14_09_18_16_28_16_2014-09-setembro. pdf. Acesso em: 29 de setembro de 2015 .

COSTA, M.H.; PIRES, G.F. Effects of Amazon and Central Brazil deforestation scenarios on the duration of the dry season in the arc of deforestation. International Journal of Climatology, v. 30, p. 1970-1979, 2010.

CUNHA, J.E.B.L.; RUFINO, L.A.A.; BERNADO, B.S.; CHAVES, L.B. Dinâmica da cobertura vegetal para a Bacia de São João do Rio do Peixe, PB, utilizando-se sensoriamento remoto. Revista Brasileira de Engenharia Agrícola e Ambiental, v. 16, n. 5, p. 539-548, 2012.

da SILVA, B.B.; WILCOX, B.P.; da SILVA, V.P.R.; MONTENEGRO, S.M.G.L.; OLIVEIRA, L.M.M.; Changes to the energy budget and evapotranspiration following conversion of tropical Savannas to agricultural lands in São Paulo State, Brazil. Ecohydrology, v. 9, n. 7, p. 1272-1283, 2014.

DANELICHEN, V.H.M.; BIUDES, M.S.; SOUZA, M.C.; MACHADO, N.G.; CURADO, L.F.A. Soil Thermal Diffusivity of a Gleyic Solonetz Soil Estimated by Different Methods in the Brazilian Pantanal. Open Journal of Soil Science, v. 3, n. 1, p. 15-22, 2013.

DIAS, L.C.P.; MACEDO, M.N.; COSTA, M.H.; COE, M.T.; NEILL, C. Effects of land cover change on evapotranspiration and streamflow of small catchments in the Upper Xingu River Basin, Central Brazil. Journal of Hydrology: Regional Studies, v. 4, p. 108-122, 2015

dos SANTOS, C.A.; do NASCIMENTO, R.L.; RAO, T.V.R. Net radiation estimation under pasture and forest in Rondônia, Brazil, with TM Landsat 5 images. Atmósfera, v. 24, n. 4, p. 435-446, 2011

DUARTE, H.F.; DIAS, N.L.; MAGGIOTTO, S.R. Assessing daytime downward longwave radiation estimates for clear and cloud skies in Southern Brazil. Agricultural and Forest Meteorology, v. 139, p. 171-181, 2006.

FAUSTO, M.A.; MACHADO, N.G.; NOGUEIRA J.S.; BIUDES, M.S. Net radiation estimated by remote sensing in Cerrado areas in the Upper Paraguay River Basin. Journal of Applied Remote Sensing, v. 8, n. 1 p. 1-17, 2014. 
FEARNSIDE, P.M. Deforestation control in Mato Grosso: a newmodel for slowing the loss of Brazil's Amazon forest. Ambio, v. 32, n. 5, p. 343-345, 2003.

FISHER, R.A.; WILLIAMS, M.; RUIVO, M.L.; DE COSTA, A.L.; MEIR, P. Evaluating climatic and soil water controls on evapotranspiration at two Amazonian rainforest sites. Agricultural and Forest Meteorology, v. 148, p. 850-861, 2008.

FU, B.; BURGHER, I. Riparian vegetation NDVI dynamics and its relationship with climate, surface water and groundwater. Journal of Arid Environments, v. 113, p. 59-68, 2015.

HASLER, N.; AVISSAR, R. What controls evapotranspiration in the Amazon Basin? Journal of Hydrometeorology, v. 8, p. 380-395, 2007.

IBGE, Instituto Brasileiro de Geografia e Estatística. Levantamento sistemático da produção agrícola: pesquisa mensal de previsão e acompanhamento das safras agrícolas no ano civil, agosto 2014. Brasília, DF, 2014. 86p. Disponível em: http://www.ibge.gov.br/home/estatistica/indicadores/ agropecuaria/lspa/lspa_201202.pdf. Acesso em: 29 de setembro de 2015.

LATHILLIÈRE, M.J.; JOHNSON, M.S.; DONNER, S.D. Water use by terrestrial ecosystems: temporal variability in rainforest and agricultural contributions to evapotranspiration in Mato Grosso, Brazil. Environmental Research Letters, v. 7, p. 1-12, 2012.

LIANG, S.; SHUEY, C.J.; RUSS, A.L.; FANG, H.; CHEN, M.; WALTHALL, C.L.; DAUGHTRY, C.S.T.; HUNT JR., R. Narrowband to broadband conversions of land surface albedo: I. Algorithms. Remote Sensing of Environment, v. 84 , p. $25-44,2000$.
LIANG, S.; SHUEY, C.J.; RUSS, A.L.; FANG, H.; CHEN, M. et al. Narrowband to broadband conversions of land surface albedo: II. Validation. Remote Sensing of Environment, v. 84, p. 25-44, 2002.

MATINS, A.L.; CUNHA, C.R.; PEREIRA, V.M.R.; DANELICHEN, V.H.M.; MACHADO, N.G. et al. Mudanças em índices biofísicos devido à alteração da cobertura do solo em área nativa de Cerrado em Mato Grosso. Ciência e Natura, v. 37, n. 4, p. 152-159, 2015.

Ministério do Meio Ambiente (MMA). Relatório Técnico de Monitoramento de Desmatamento do Bioma Cerrado de 2009 a 2010. 2011. Disponível em: http://www.mma.gov. br/estruturas/sbf_chm_rbbio/_arquivos/relatoriofinal_cerra do_2010_final_72_1.pdf . Acesso em 25 de dezemb̄o de 2015 .

MYERS, N.; MITTERMEIER, R.A.; MITTERMEIER, C.G.; FONSECA, G.A.B.; KENT, J. Biodiversity hotspots for conservation priorities. Nature, v. 403, p. 853-858, 2000.

MENDONÇA, R.C.; FELFI LI, J.M.; WALTER, B.M.T.; SILVA JÚNIOR, M.C.; REZENDE, A.V. et al. Flora Vascular do Cerrado. pp. 289-556. In: SANO, S.M.; ALMEIDA, S.P. (eds). Cerrado: ambiente e flora. Planaltina, EMBRAPACPAC.

SHEIL, D.; MURDIYARSO, D. How forests attract rain: an examination of a new hypothesis. BioScience, v. 59, n. 4, p. 341-347, 2009.

VALOR, E.; CASELLES, V. Mapping land surface emissivity from NDVI: Application to European, African, and South American areas. Remote Sensing of Environment, v. 57, p. 167-184, 1996.

This is an Open Access article distributed under the terms of the Creative Commons Attribution Non-Commercial License which permits unrestricted non-commercial use, distribution, and reproduction in any medium provided the original work is properly cited. 